\title{
Interleukin-17 promotes nitric oxide-dependent expression of PD-L1 in mesenchymal stem cells
}

Shijia Wang ${ }^{1}$, Guan Wang ${ }^{2}$, Liying Zhang ${ }^{3}$, Fengying Li ${ }^{2}$, Keli Liu ${ }^{2}$, Ying Wang ${ }^{2 *}$, Yufang Shi ${ }^{1,2,3^{*}}$ and Kai Cao ${ }^{2^{*}}$

\begin{abstract}
Background: Interleukin-17A (IL-17) is an evolutionary conserved cytokine and best known for its role in boosting immune response. However, recent clinical researches showed that abundant IL-17 in tumor microenvironment was often associated with poor prognosis and reduced cytotoxic T cell infiltration. These contradictory phenomena suggest that IL-17 may have unique target cells in tumor microenvironment which switch its biological consequences from pro-inflammatory to anti-inflammatory. Mesenchymal stem/stromal cells (MSCs) are a major component of the tumor microenvironment. Upon cytokine stimulation, MSCs can express a plenary of inhibitory molecules, playing a critical role in tumor development and progression. Therefore, we aim to investigate the role of IL-17 in MSC-mediated immunosuppression.

Results: We found IFNy and TNFa, two major cytokines in tumor microenvironment, could induce programmed death-ligand 1 (PD-L1) expression in MSCs. Interestingly, IL-17 has a synergistic effect with IFNY and TNFa in elevating PD-L1 expression in MSCs. The presence of IL-17 empowered MSCs with strong immunosuppression abilities and enabled MSCs to promote tumor progression in a PD-L1 dependent manner. The upregulated PD-L1 expression in MSCs was due to the accumulation of nitric oxide (NO). On one hand, NO donor could mimic the effects of IL-17 on MSCs; on the other hand, IL-17 failed to enhance PD-L1 expression in inducible nitric oxide synthase (iNOS) deficient MSCs or with iNOS inhibitor presence.

Conclusions: Our study demonstrates that IL-17 can significantly increase the expression of PD-L1 by MSCs through iNOS induction. This IL-17-MSCs-PD-L1 axis shapes the immunosuppressive tumor microenvironment and facilitates tumor progression.
\end{abstract}

Keywords: Mesenchymal stem/stromal cells, Programmed death-ligand 1, Interleukin-17, Nitric oxide, Tumor microenvironment

*Correspondence: yingwang@sibs.ac.cn; yufangshi@sibs.ac.cn; kai. cao@glasgow.ac.uk

${ }^{1}$ Key Laboratory of Stem Cell Biology, Shanghai Jiao Tong University School of Medicine (SJTUSM) \& Shanghai Institutes for Biological Sciences (SIBS), Academy of Sciences (CAS), Shanghai 200031, China

${ }^{2}$ CAS Key Laboratory of Tissue Microenvironment and Tumor, Shanghai Institute of Nutrition and Health, Shanghai Institutes for Biological

Sciences, University of Chinese Academy of Sciences, Chinese Academy of Sciences, Shanghai 200031, China

Full list of author information is available at the end of the article

\section{Background}

Interleukin-17A (IL-17) is the first member discovered in the IL-17 cytokine family [1]. It is primarily produced by Th17 cells during immune responses and exerts strong pro-inflammatory functions in infectious, inflammation and autoimmune diseases $[2,3]$. The great success of tumor immunotherapies highlights the roles of immune responses in tumor progression, in which the role of IL-17 has also drawn much attention $[4,5]$. Th17 cells have been found in various types of human cancers 
and different experimental animal tumor models [6-8]. Despite its superior abilities in augmenting immune response, both clinical studies and animal models suggests Th17-related cytokines, such as IL-17 and IL-23, can promote tumor progression $[9,10]$. Currently, the tumor promoting effects of IL-17 are regarded as its effects on tumor cells and vascular endothelial cells [11, 12]. However, the role of other components of tumor stroma in IL-17-mediated modulation of tumor growth remains obscure.

In solid tumors, tumor stroma provides fertile soil for tumor growth and thus plays important role in facilitating tumor development [13]. Mesenchymal stem cells (MSCs) are one of the major components of tumor stroma $[14,15]$. MSCs are highly plastic cells and often converted to tumor associated-MSCs in tumor microenvironment, and thus obtain the abilities to promote tumor progression $[14,16]$. Besides directly promoting tumor growth and angiogenesis through their secretome [14, 17], MSCs could indirectly facilitate tumor development through shaping an immunosuppressive microenvironment to help tumor escape the surveillance of immune system [18]. Upon the stimulation of proinflammatory cytokines, MSCs can potently suppress lymphocytes proliferation through the concerted action of chemokines and inhibitory molecules [19, 20]. Our previous works showed that tumor-associate MSCs dramatically enhanced tumor growth by recruiting suppressive immune cells to the tumor microenvironment [15, $21,22]$. MSCs could even reprogram the responsiveness of macrophages and conferred their anti-inflammatory abilities [23]. Importantly, the effects of MSCs on immune system are dependent on the cytokine milieu [18]. It is worth noting that MSCs express IL-17 receptor [24]. Therefore, IL-17 could have a role in regulating tumor associated MSCs.

Programmed death-ligand 1 (PD-L1), also known as $\mathrm{CD} 274$, is a B7 family protein mainly expressed on antigen presenting cells, such as macrophages and monocytes $[25,26]$. PD-L1 ligation with programmed death 1 (PD-1) on activated $\mathrm{T}$ cells and $\mathrm{B}$ cells are regard as negative feedback to fine tune inflammation [27]. Dysregulated PD-L1-PD-1 pathway often leads to various inflammatory diseases, including GvHD, hepatitis, and more importantly, cancer [28, 29]. Tumor cells are found to escape immune surveillance through upregulating PD-L1 expression on their surface [30, 31]. Epidemiological investigations have proved the correlation between PD-L1 expression and tumor prognosis and great endeavor are spent to decipher the molecular mechanism of PD-L1-mediate immune suppressive pathway [25, 32]. The breakthrough in PD-L1 biology brought PD-L1-based tumor therapy to the spotlight and PD-L1 neutralizing antibodies have been approved by Food and Drug Administration for the treatment of various cancers [32]. Besides its expression on tumor cells, PD-L1 is also expressed by MSCs to exert their immunosuppressive properties [33-35]. Notably, positive correlation between IL-17 and PD-L1-expressing tumor stromal cells has been demonstrated [36]. Based on this information, it is possible that IL-17 can enhance PD-L1 expression on MSCs to promote tumor growth.

In this study, we found that IL-17 combined with IFN $\gamma$ and TNF $\alpha$ dramatically enhanced PD-L1 expression of MSCs, compared to IFN $\gamma$ and TNF $\alpha$ stimulation. Interestingly, we found this synergistic induction of PD-L1 was dependent on nitric oxide (NO) production by MSCs, since genetic deletion of inducible nitric oxide synthase (iNOS) and iNOS chemical inhibitor abolished this effect. Furthermore, NO donor could act as the substitute of IL-17 to promote PD-L1 expression through STAT3 pathway. To verify the biological consequence of this effect in vivo, we injected IFNY and TNFa primed MSCs with or without IL-17 co-stimulation together with B16 melanoma to mice. We found that IL-17 significantly enhanced the tumor promoting effects of MSCs. Furthermore, this effect can be abrogated by the administration of PD-L1 neutralizing antibody, confirming the critical role of PD-L1 in this process. Our findings reveal a novel mechanism underlying the tumor promotive property of IL-17, which is through inducing PD-L1 expression on MSCs.

\section{Results}

\section{IL-17 enhanced the PD-L1 expression on MSCs}

PD-L1 is one of the most potent immunosuppressive molecules and plays an important role in tumor development. Clinical applications of PD-L1 neutralizing antibodies have achieved great success in various cancers [37]. MSCs, a major component of the tumor microenvironment, are reported to express PD-L1 upon cytokine stimulation [34, 38]. Additionally, in the peritumoral stroma of hepatocellular carcinoma (HCC) patients, there is a positive correlation between IL-17 producing cells and PD-L1 expressing cells, indicating a possible relationship between IL-17 and stromal PD-L1 expression [36]. To test this hypothesis, we stimulated MSCs with IL-17 in vitro. However, IL-17 could not directly induce PD-L1 expression on MSCs (Fig. 1a). Since another important function of IL-17 is to synergize with other cytokines to upregulate gene expression $[12,39]$. We tested whether IL-17 could further enhance the PD-L1 expression induced by IFN $\gamma$ and TNF $\alpha$ [34, 40]. We found that in this scenario, IL-17 dramatically enhanced PD-L1 expression (Fig. 1a). Furthermore, the effect of IL-17 was not observed at $12 \mathrm{~h}$, suggesting that 
a

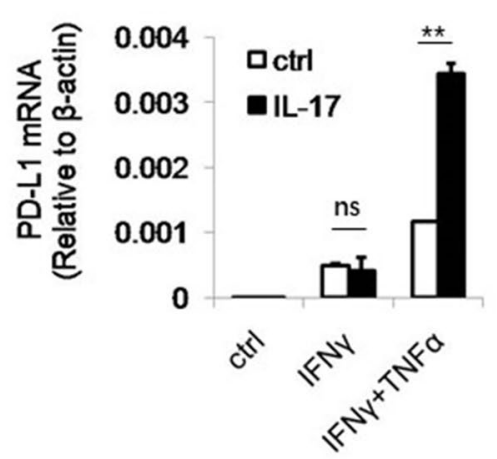

C

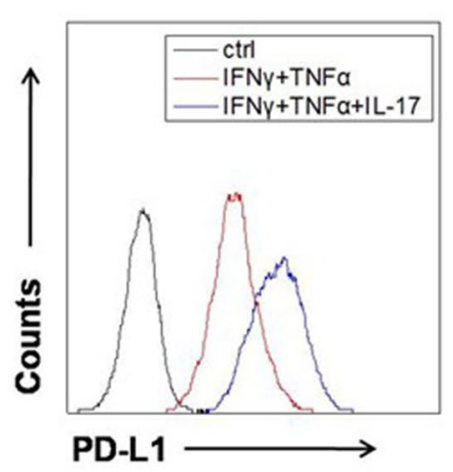

b

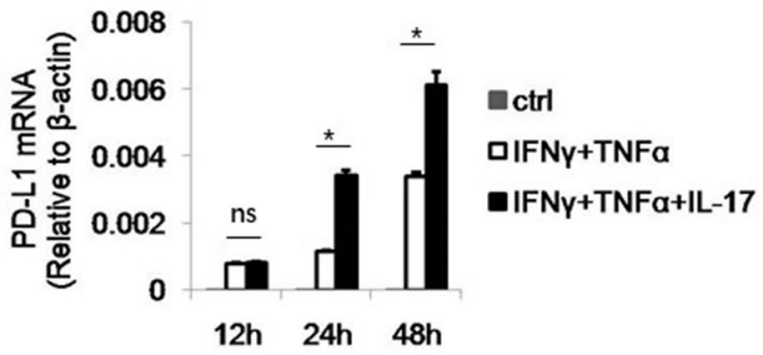

d

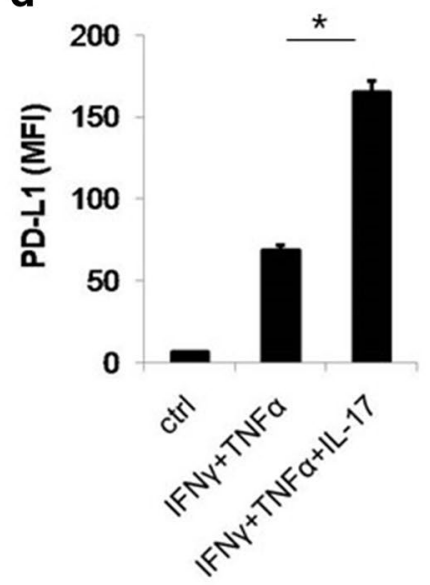

Fig. 1 IL-17 synergizes with IFNy and TNFa to promote PD-L1 expression on MSCs. a C57BL/6 MSCs were stimulated by indicated combination of cytokines. The concentration of three cytokines was $10 \mathrm{ng} / \mathrm{ml}$ each, for this and all other figures. After $24 \mathrm{~h}$, cells were harvested and prepared for quantitation of PD-L1 by real-time PCR. b C57BL/6 MSCs were treated with different combination of cytokines. Cells were collected at the indicated time points for assay of FPD-L1 mRNA by real-time PCR. c C57BL/6 MSCs were treated as in $\mathbf{b}$. After $48 \mathrm{~h}$, cells were harvested and the expression of PD-L1 was determined by flow cytometry. $\mathbf{d}$ The statistical results of three separate experiments described in $\mathbf{c}$

the PD-L1 upregulation might be a secondary effect of IL-17 (Fig. 1b). To confirm this synergized effect of IL-17, we next examined the expression of PD-L1 on protein level. As expect, IL-17 combined with IFN $\gamma$ and TNF $\alpha$ also elevated PD-L1 protein level on the surface of MSCs (Fig. 1c, d). Taken together, IL-17 and inflammatory cytokines, acting in concert, can promote the expression of PD-L1 on MSCs.

\section{Nitric oxide is indispensable for the synergistic effect of IL-17 on PD-L1 expression}

As we demonstrated, IL-17 did not show synergistic effect on PD-L1 expression before $12 \mathrm{~h}$. Therefore, we wonder if IL-17 may act through other molecules to induce PD-L1 expression. Our previous studies have shown that IL-17 can similarly synergize with IFN $\gamma$ and TNF $\alpha$ to induce the expression of iNOS and the production of
NO [24], which is confirmed in our experiment (Fig. 2a, b). In addition, we found that the production of NO by MSCs is not detectable until $12 \mathrm{~h}$ (Fig. 2c). Therefore, we considered $\mathrm{NO}$ as the candidate of the mediator between IL-17 and enhanced PD-L1 expression. To identify the role of NO in this process, we employed iNOS inhibitor S-methylisothiourea (SMT) and iNOS knockout MSCs. It is observed that NO production by wild type (WT) MSCs was completely abolished by SMT treatment and $\mathrm{NO}$ was barely detectable in the supernatant of $\mathrm{iNOS}^{-/-}$ MSCs (Fig. 2d).

Furthermore, both mRNA and protein level of PD-L1 showed that the synergistic effect of IL-17 was abolished by SMT or iNOS deficiency, suggesting NO is indispensable for IL-17 to promote PD-L1 expression (Fig. 2eg). In iNOS $^{-1-}$ MSCs, the induction of PD-L1 by IL-17 was also abrogated, confirming the role of NO in IL-17 

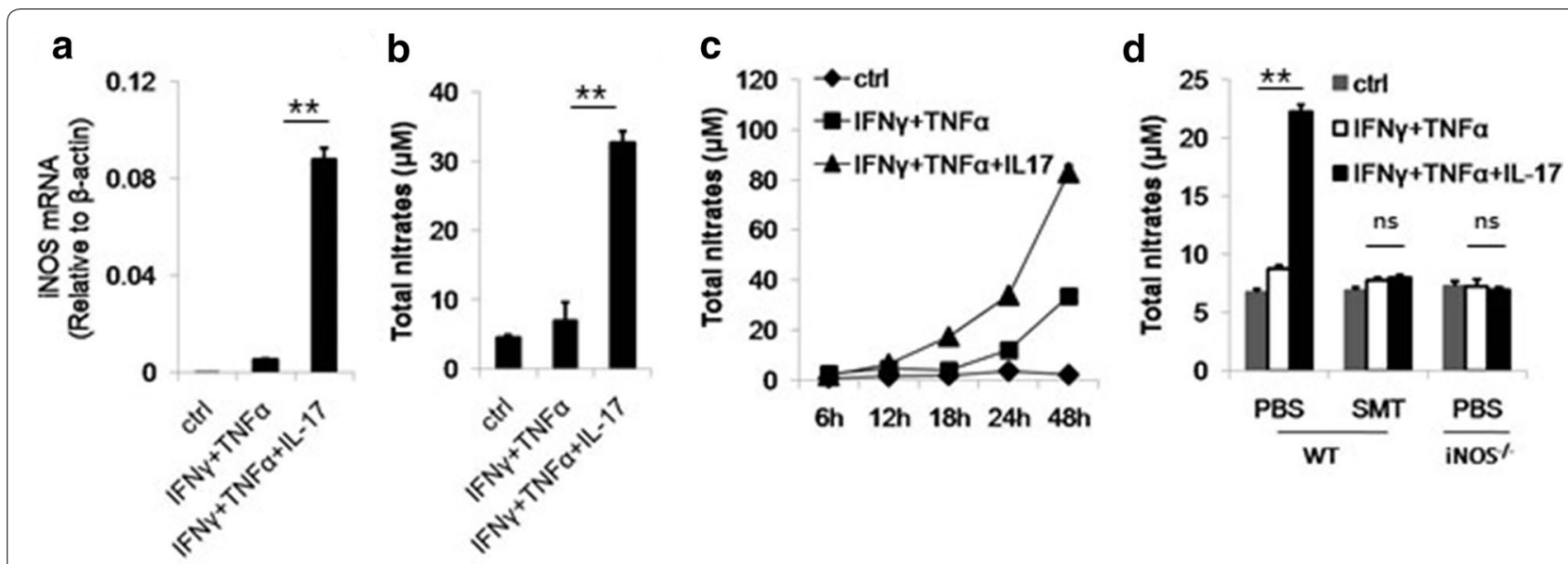

e

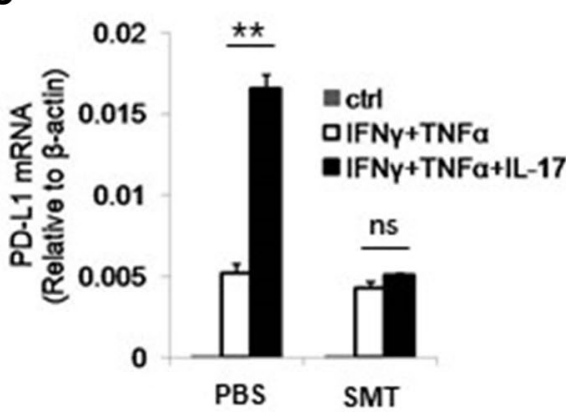

h

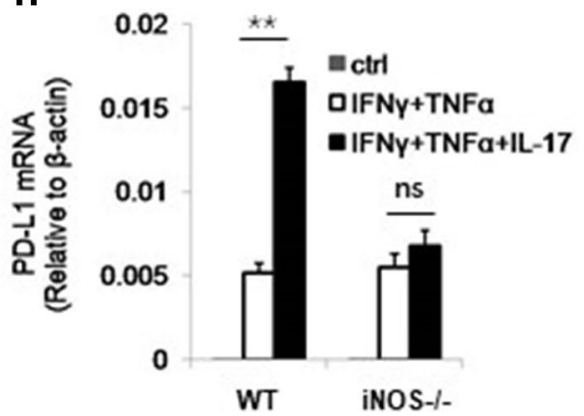

f

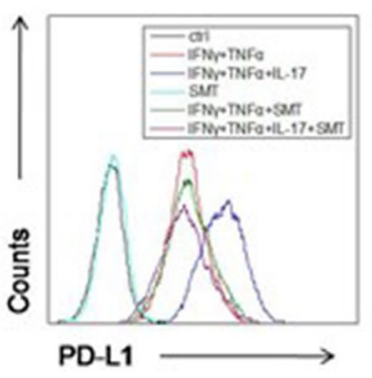

i

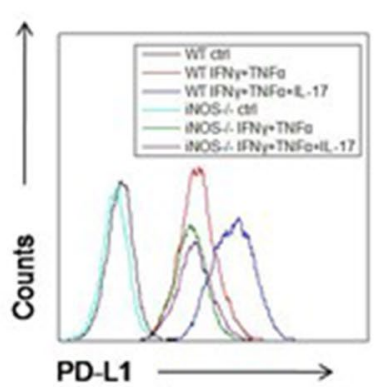

g

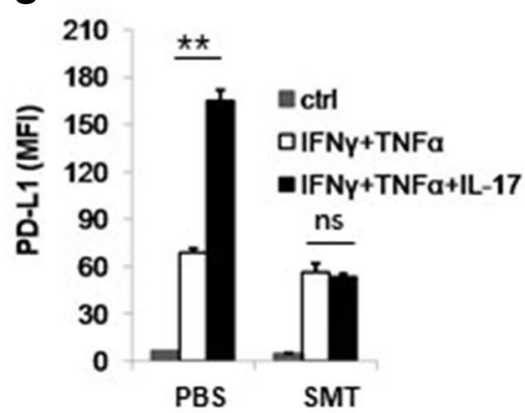

j

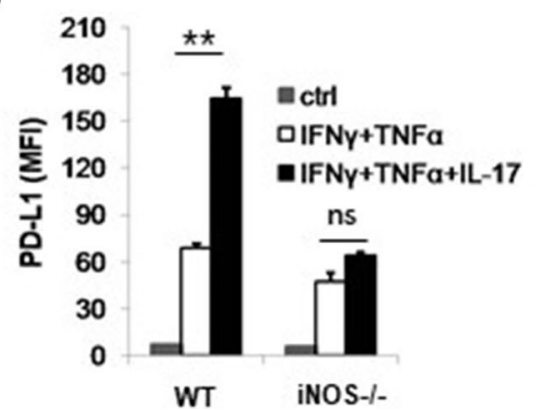

Fig. 2 IL-17 enhances the expression of PD-L1 through NO. a C57BL/6 MSCs were treated by indicated cytokines for $24 \mathrm{~h}$ and then collected for the measurement of NO mRNA by real-time PCR. b, c C57BL/6 MSCs were treated as in A and after $24 \mathrm{~h}$ (b) or indicated time points (c), supernatant were collected for the assay of NO concentration by Griess reagent. $\mathbf{d}$ WT MSCs (C57BL/6 MSCs) and iNOS ${ }^{-/-}$MSCs were treated by cytokines and SMT (20 mM) for $24 \mathrm{~h}$ and supernatant were collected for the measurement of NO. e, f C57BL/6 MSCs were treated by cytokines with or without SMT. After $24 \mathrm{~h}$, cells were collected for the assay of PD-L1 mRNA by real-time PCR. After $48 \mathrm{~h}$, cells were collected for the assay of PD-L1 protein by

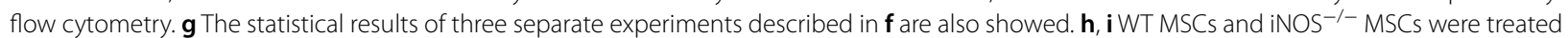
with cytokines. Cells were collected for the measurement of PD-L1 mRNA and protein after $24 \mathrm{~h}$ and $48 \mathrm{~h}$, respectively. $\mathbf{j}$ The statistical results of three separate experiments described in $\mathbf{i}$ are also showed

mediated PD-L1 enhancement (Fig. 2h-j). To further validate this point, we applied $S$-Nitroso- $N$-acetyl-DLpenicillamine (SNAP) as NO donor into MSCs culture medium. We found the inhibitory effect of SMT on PD-L1 induction was reversed on both mRNA and protein level (Fig. 3a-c). Moreover, SNAP has the similar effects on iNOS ${ }^{-1-}$ MSCs (Fig. 3d-f). Taken together, we demonstrated that NO mediated the promotive effect of IL-17 on PD-L1 expression of MSCs. These results also raised the possibility that NO could be the substitute of IL-17 to promote PD-L1 expression on MSCs. To test this hypothesis, we added SNAP with IFN $\gamma$ and TNF $\alpha$ 

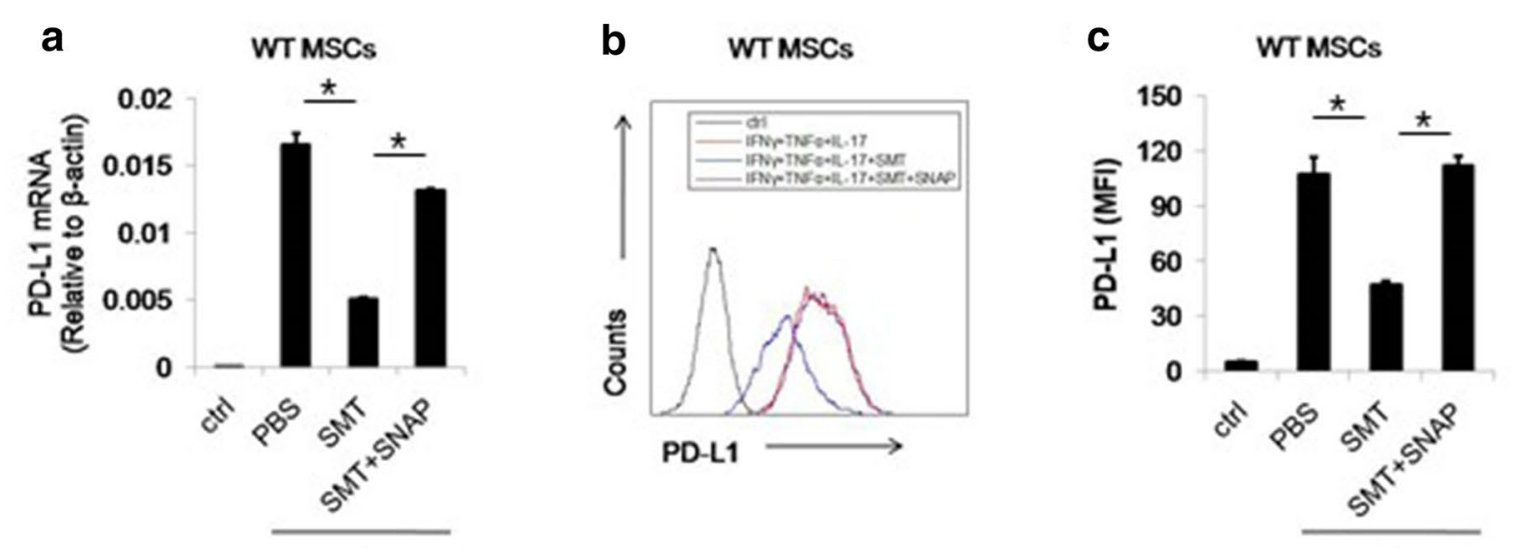

IFNY+TNFa+IL-17

IFNY+TNFa+IL-17
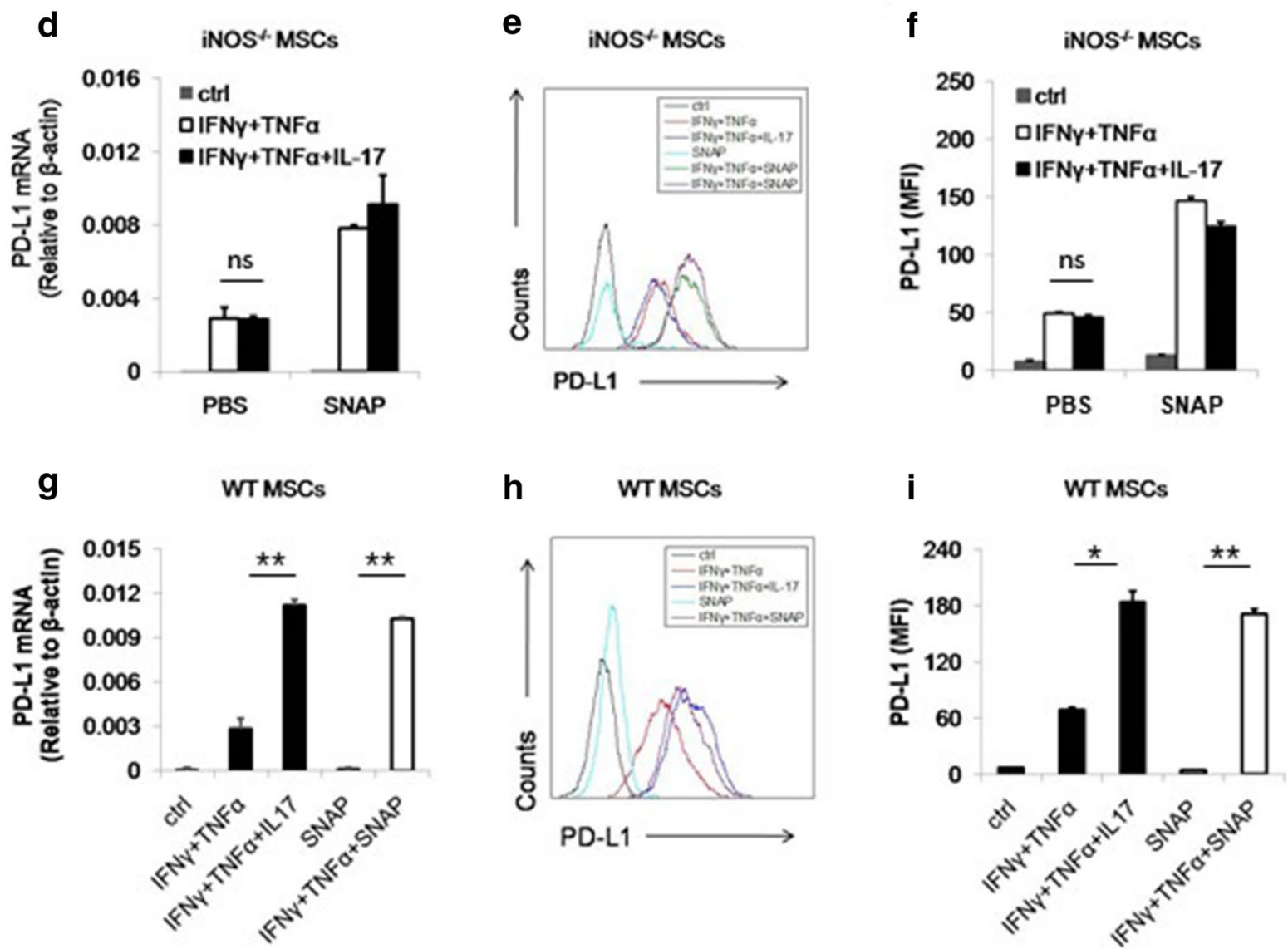

Fig. 3 NO donor can reinstall the expression of PD-L1 on MSCs. $\mathbf{a}$, b WT MSCs were treated by IFNy, TNFa and IL-17 with or without SMT and SNAP $(1 \mathrm{mM})$. The expression of PD-L1 mRNA and protein was then measured after $24 \mathrm{~h}$ and $48 \mathrm{~h}$, respectively. $\mathbf{c}$ The statistical results of three separate experiments described in B are also showed. $\mathbf{d}$, e iNOS ${ }^{-1-}$ MSCs were stimulated by cytokines in the presence or absence of SNAP. The expression of PD-L1 mRNA and protein was then measured after $24 \mathrm{~h}$ and $48 \mathrm{~h}$, respectively. $\mathbf{f}$ The statistical results of three separate experiments described in e are also showed. $\mathbf{g}, \mathbf{h}$ WT MSCs were treated with indicated combinations of cytokines. After $24 \mathrm{~h}$ and $48 \mathrm{~h}$, the expression of PD-L1 mRNA and protein was then measured, respectively. $\mathbf{i}$ The statistical results of three separate experiments described in $\mathbf{h}$ are also showed 
in culture medium of MSCs. Interestingly, we found that the combination of these three molecules can stimulate the expression of PD-L1 to the same level of IFN $\gamma$, TNF $\alpha$ and IL-17 treated MSCs (Fig. 3g-i). Notably, SNAP alone could not enhance the expression of PD-L1, indicating certain signaling pathways activated by IFN $\gamma$ and TNF $\alpha$ were required for NO to exert its function on MSCs. Thus, we concluded that IL-17-induced NO acts in concert with IFN $\gamma$ and TNF $\alpha$ signal to promote the expression of PD-L1 on MSCs.

\section{The effect of IL-17 on PD-L1 expression is dependent of NO-activated STAT3 phosphorylation}

To elucidate the molecular mechanism by which IL-17 promote PD-L1 expression on MSCs, we test the effect of IL-17 on transcription factors responsible for PD-L1 expression. NF-kB and STAT3 are best characterized transcriptional factors which were reported to regulate PD-L1 [41-43]. We thus examined the activity of NF-kB and STAT3 upon IL-17 stimulation. We found that although p65 phosphorylation was increased upon the stimulation of IFN $\gamma$ and TNF $\alpha$, the addition of IL-17 did not further strengthen this signal. On the other hand, IL-17 dramatically enhanced the phosphorylation of STAT3, indicating NO may act through STAT3 to promote PD-L1 expression (Fig. 4a). To further validate this result, we examined whether $\mathrm{NO}$ donor also regulate PD-L1 in a STAT3 dependent manner. We found in the presence of IFN $\gamma$ and TNF $\alpha$, SNAP enhanced the signal transduction by STAT3 (Fig. 4b). Interestingly, SMT could also induce STAT3 phosphorylation while failed to boost PD-L1 expression. A possible explanation which reconcile the contradictory observations is that p-STAT3 signaling is indispensable but not sufficient to induce PD-L1 expression. NO may also corporate with p-STAT3 signaling to boost PD-L1 expression through other signaling pathways in MSCs. Further investigations are required to fully address this important issue.

SNAP alone can also activate STAT3, however, it cannot induce PD-L1. To prove the cause effect between

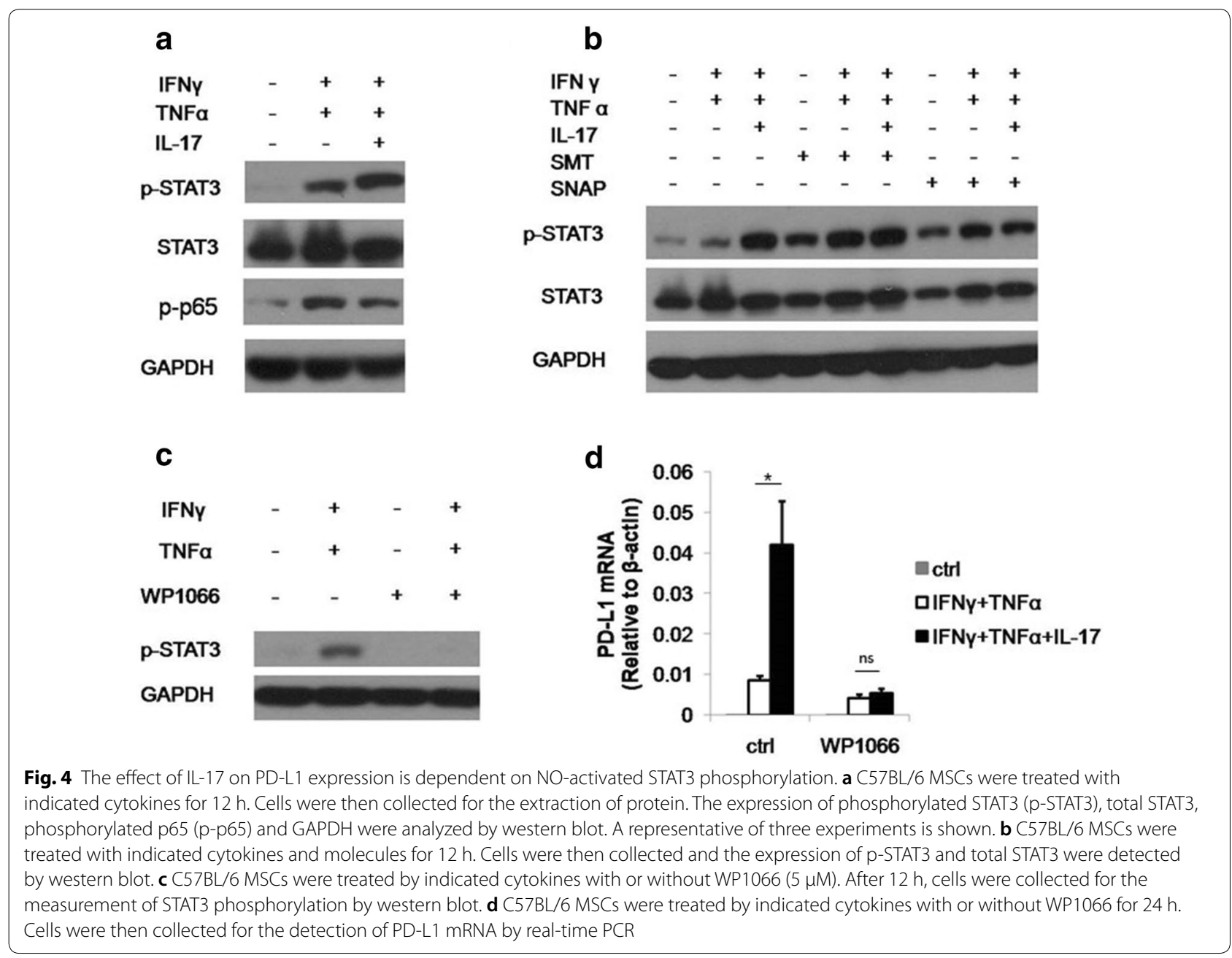


enhanced STAT3 signaling and upregulated PD-L1 expression, we employed STAT3 inhibitor WP1066 which can efficiently suppress the activity of STAT3 pathway (Fig. 4c). We found that when the STAT3 phosphorylation was inhibited, the expression of PD-L1 was also dramatically depressed (Fig. 4d). These experiments showed that IL-17 promoted PD-L1 expression through NO-activated STAT3 pathway.

\section{IL-17 pretreated MSCs promote tumor growth through PD-L1}

Our in vitro data showed IL-17 could dramatically enhanced PD-L1 expression on MSCs. Considering the importance of PD-L1 in tumor progression and the presence of MSCs in the tumor microenvironment, it is possible that IL-17 could promote tumor growth through upregulating PD-L1 expression on MSCs. To test whether IL-17 shapes the tumor promoting effects of MSCs, we established melanoma model through the administration of B16F0 tumor cells together with cytokine-pretreated MSCs, and then compared the effect of MSCs with or without IL-17 on tumor growth. We found that the administration of MSCs with IL-17 pretreatment significantly promoted melanoma growth (Fig. 5a). To verify whether the tumor promoting effects of IL-17 primed MSCs are dependent on PD-L1, we employed PD-L1 neutralizing antibody to block ligation site of PD-L1 (Fig. 5b). In this condition, we found that IL-17 primed MSCs can no longer promote tumor growth in vivo (Fig. 5c). To further confirm this result, we also administrated PD-L1 antibodies to tumor bearing mice. As expected, the effect of IL-17 pretreated MSCs on tumor growth is completely inhibited (Fig. 5d). Collectively, our data suggested that IL-17 can act through MSCs to promote melanoma growth.

\section{Discussion}

In the last decade, it has been established that MSCs participate in tumor development and metastasis through shaping tumor microenvironment [14]. Substantial evidence showed that MSCs express a plenary of molecules to exert their tumor promoting effects, either by suppressing cytotoxic immune responses or promoting the recruitment of immunosuppressive macrophages [22]. We found that IL-17 can synergize with IFN $\gamma$ and TNF $\alpha$ to promote PD-L1 expression of MSCs. IFN $\gamma$, TNF $\alpha$ and IL17 commonly function as pro-inflammatory cytokines. IFN $\gamma$ and $\mathrm{TNF} \alpha$ are mainly secreted by immune cells, such as lymphocytes and NK cells, and act as an important inducer of various cytokine and chemokine genes [44, 45]. Ironically, these pro-inflammatory cytokines are also present in immune paralysis tumor microenvironment. Our

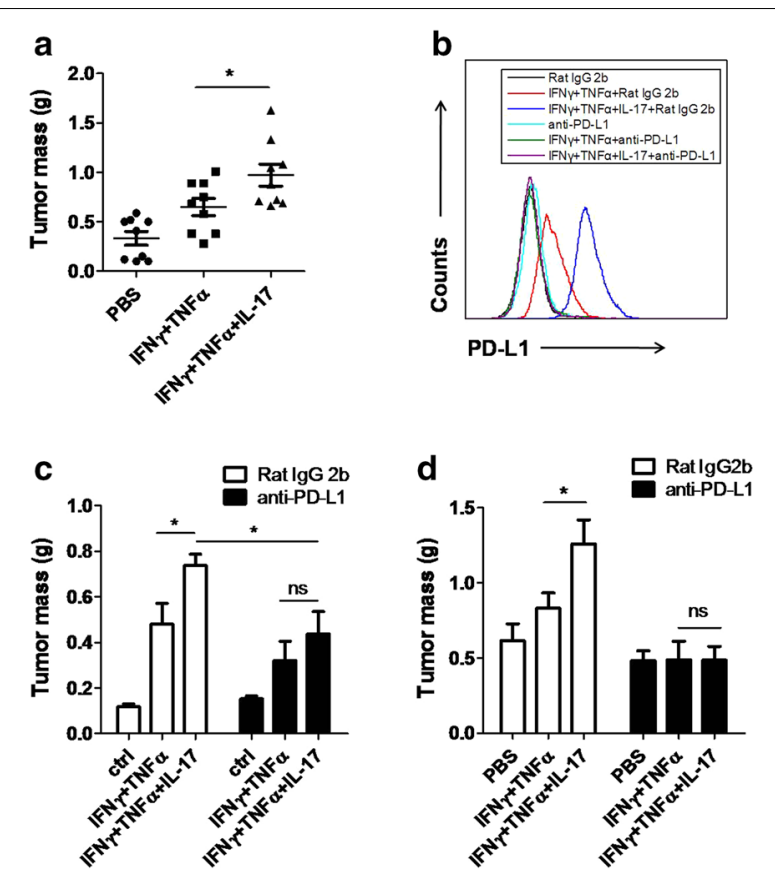

Fig. 5 IL-17-pretreated MSCs promote melanoma growth through enhancing PD-L1 expression on MSCs. a C57BL/6 MSCs were treated by PBS, or IFN $\gamma$ and TNFa, or IFN $\gamma$, TNFa and IL-17 for $48 \mathrm{~h}$. Pretreated MSCs $\left(1 \times 10^{5}\right)$ were then collected and injected subcutaneously together with B16F0 cells $\left(2.5 \times 10^{5}\right)$ on C57BL/6 mice $(n=9$ each). On day 16 , mice were euthanized and the resultant tumors were excised and weighed. $\mathbf{b}$ C57BL/6 MSCs were treated as in a with or without PD-L1 antibody $(10 \mu \mathrm{g} / \mathrm{ml})$. After $48 \mathrm{~h}$, cells were collected for the detection of PD-L1 by flow cytometry. c C57BL/6 MSCs collected in $\mathbf{b}$ were then injected with B16 cells as in $\mathbf{a}(\mathrm{n}=5$ each). On day 16 , mice were euthanized and the resultant tumors were excised and weighed. $\mathbf{d}$ C57BL/6 MSCs $\left(1 \times 10^{5}\right)$ and B16 cells $\left(2.5 \times 10^{5}\right)$ were injected as in a. On day 1,4 and 7, PD-L1 antibodies $(10 \mu \mathrm{g} / \mathrm{g})$ were administrated intraperitoneously ( $n=6$ each). On day 16 , mice were euthanized and the resultant tumors were excised and weighed. Statistical analysis was performed using the Student's t-test. ${ }^{*} p<0.05$, ns (not significant)

previous studies showed that TNF $\alpha$ treated MSCs can express chemokines to recruit M2-like macrophages to promote tumor growth, suggesting these generally proinflammatory cytokines could induce immunosuppression through MSCs. In the current study, we showed this is also true with IL-17. Therefore, it is important to interpret the biological effects of cytokines in the context of MSCs.

In our study, we have shown that NO is responsible for the enhanced PD-L1 expression on MSCs, since genetic deletion of iNOS gene and iNOS inhibitor could suppress the PD-L1 expression. Notably, the suppression of NO production did not completely inhibit the expression of PD-L1 as we expected, but down to the same level of that as treated with IFN $\gamma$ and TNF $\alpha$. 
This indicates that PD-L1 gene expression in MSCs is induced by IFN $\gamma$ and TNF $\alpha$ and further enhanced by $\mathrm{NO}$ accumulation. Without the signal of IFN $\gamma$ and TNF $\alpha$, NO alone could not induce PD-L1 expression. This conclusion is further verified by the fact that NO donor alone showed no effect on PD-L1 expression, while together with IFN $\gamma$ and TNF $\alpha$, NO can act as the substitute of IL-17 to enhance the expression of PD-L1.

$\mathrm{NO}$ is reported to regulate the expression of various cytokines, growth factors and chemokines, including IL-6, IL-10, IFN $\gamma$, TGF $\beta$, M-CSF, VEGF and MCP-1, which are important in tumor growth and metastasis [46]. Here, we reported that NO induced expression of PD-L1 on MSCs through activating STAT3. However, detail studies are still required to fully illustrated how NO promote STAT3 phosphorylation. The effect of $\mathrm{NO}$ on protein is often achieved through introducing a nitro group $\left(-\mathrm{NO}_{2}\right)$ to amino acids, mainly tyrosines [47]. This tyrosine nitration process will result in directly gain or inhibition of protein function, or indirectly influence the phosphorylation process to further regulate protein function. Therefore, it is possible that in MSCs, NO can result in the nitration of STAT3, which in turn increases the phosphorylation of STAT3. This study would also provide novel information of NO-mediated inhibition of immune responses.

Our studies showed that IL-17 pre-treated MSCs can promote tumor growth through enhanced PD-L1 expression. PD-L1 exerts its effect mainly through directly inhibiting lymphocyte activation and proliferation, especially CD8+ T cells. It is worth investigating that how these PD-L1 expressing MSCs regulates tumor microenvironment. It is reported that MSCs often promote tumor progression through Treg induction. An important function of PD-L1 is to upregulate Treg. Therefore, the Treg induced by tumor-associated MSCs may due to its PD-L1 expression. This merits further investigation.

In conclusion, the results presented here suggest that IL-17 can synergize with IFN $\gamma$ and TNF $\alpha$ to promote PD-L1 expression on MSCs. Enhanced expression of PD-L1 on MSCs within tumor microenvironment will result in increased tumor growth. Thus, we have proposed a new mechanism in which IL-17 act through MSCs to promote tumor growth, which enriched our understanding of the relationship between IL-17 and tumor growth and due to the important role of PD-L1 in controlling immune responses, our study also provided novel information for the application of IL-17 treated MSCs in not only cancer, but also inflammatory diseases.

\section{Methods}

\section{Animals}

C57BL/6 mice were purchased from the Shanghai Laboratory Animal Center of Chinese Academy of Sciences, Shanghai, China, and maintained under specific pathogen-free (SPF) conditions. $\mathrm{iNOS}^{-1-}$ mice were obtained from Jackson Laboratory (Bar Harbor, ME, USA). Mice were housed in the Vivarium of Shanghai Jiao Tong University School of Medicine. All procedures were approved by the Institutional Animal Care and Use Committee of the Institute of Health Sciences, Shanghai Institutes for Biological Sciences of Chinese Academy of Sciences. Animals were matched for age and gender in each experiment.

\section{Antibodies and reagents}

$S$-nitroso- $N$-acetyl-penicillamine (SNAP), PMSF and Griess reagent were from Sigma-Aldrich (St. Louis, MO, USA). Recombinant mouse IFN $\gamma$, TNF $\alpha$, IL-17A and anti-mouse CD274 (PD-L1)-APC were from eBiosciences (La Jolla, CA, USA). WP1066 was from Santa Cruz Biotechnology (Dallas, TX, USA). Antibodies against GAPDH, p-p65, p-STAT3 and STAT3 were from Cell Signaling Technology (Danvers, MA, USA). S-methylisothiourea (SMT) was from Beyotime Technoloby (Shanghai, China).

\section{Cells}

MSCs were generated using our previously described protocol. Briefly, tibia and femur bone marrow of 6-week-old wild-type or iNOS ${ }^{-1-}$ mice was harvested. Cells were cultured in DMEM medium supplemented with $10 \%$ FBS, $2 \mathrm{mM}$ glutamine, $100 \mathrm{U} / \mathrm{ml}$ penicillin, and $100 \mu \mathrm{g} / \mathrm{ml}$ streptomycin (complete medium, all from Invitrogen, Carlsbad, CA, USA). All non-adherent cells were removed after $24 \mathrm{~h}(\mathrm{hr})$, and adherent cells were maintained. Medium was changed every 3 days. To obtain MSC clones, cells at confluence were harvested and seeded into 96-well plates by limited dilution. Individual clones were then picked and expanded. These MSCs were capable of differentiating into adipocytes and osteocytes under the respective differentiation conditions. Cells were used before the 15th passage. B16F0 mouse melanoma cells were cultured in DMEM medium supplemented with $10 \% \mathrm{FBS}, 2 \mathrm{mM}$ glutamine, $100 \mathrm{U} / \mathrm{ml}$ penicillin, and $100 \mathrm{mg} / \mathrm{ml}$ streptomycin.

\section{Flow cytometric analysis}

MSCs were treated with or without different combinations of IFN $\gamma(2 \mathrm{ng} / \mathrm{ml})$, TNF $\alpha(2 \mathrm{ng} / \mathrm{ml}), \mathrm{IL}-17 \mathrm{~A}(10 \mathrm{ng} /$ $\mathrm{ml})$, SMT $(1 \mathrm{mM})$ and SNAP $(1 \mathrm{mM}) .2$ days later, cells were digested and harvested and then suspended at a 
concentration of $2 \times 10^{6}$ cells $/ \mathrm{ml}$ (in PBS with $2 \% \mathrm{FBS}$ ) and stained by anti-mouse CD274 (PD-L1) APC antibodies for $30 \mathrm{~min}$. Antibody-stained cells were washed twice with PBS. Fluorescence intensity was measured by flow cytometry (FACS Calibur, BD Immunocytometry).

\section{Real-time PCR}

Total RNA was isolated using RNAprep pure Cell/Bacteria Kit (Tiangen biotech, Beijing, China). First-strand cDNA synthesis was performed using PrimeScript ${ }^{\mathrm{TM}} \mathrm{RT}$ Master Mix (TaKaRa Biotech, Dalian, China). The levels of mRNA of genes of interest were measured by real-time PCR (7900 HT by Applied Biosystems, Foster City, CA, USA) using SYBR Green Master Mix (TaKaRa Biotech, Dalian, China). Total amount of mRNA was normalized to endogenous $\beta$-actin mRNA. Sequences of PCR primer pairs were as follows: mouse PD-L1, forward $5^{\prime}$-GCT CCAAAGGACTTGTACGTG-3' and reverse 5'-TGA TCTGAAGGGCAGCATTTC-3'; mouse iNOS, forward 5'-CAGCTGGGCTGTACAAACCTT-3' and reverse $5^{\prime}$-CATTGGAAGTGAAGCGTTTCG- ${ }^{\prime}$; mouse $\beta$-actin, forward $5^{\prime}$-CCACGAGCGGTTCCGATG-3' and reverse 5'-GCCACAGGATTCCATACCCA-3'.

\section{Detection of NO}

NO was detected using a modified Griess reagent. Briefly, $50 \mu$ supernatant was mixed with equal volumes of Griess reagent, and the concentration of total $\mathrm{NO}_{2}$ in the supernatant was detected by the Griess reaction through reading the absorbance at $540 \mathrm{~nm}$ after $15 \mathrm{~min}$.

\section{Western blotting}

Cells were washed twice with ice-cold PBS, harvested and lysed in the RIPA buffer (Millipore, Temecular, CA, USA) containing a cocktail of protease inhibitors (Roche, Natley, NJ, USA) and PMSF for $30 \mathrm{~min}$ on ice. Lysates were clarified by centrifugation at $16,000 \times g$ for $15 \mathrm{~min}$ and heated in sodium dodecyl sulfate sample buffer at $95^{\circ} \mathrm{C}$ for $10 \mathrm{~min}$. Protein concentration of the supernatant was determined by the Bradford assay (Bio-Rad, Hercules, CA, USA). Protein samples were separated on a polyacrylamide gel, and separated proteins were electroblotted onto polyvinylidene difluoride membranes. Specific proteins were revealed by mouse and rabbit antibodies against p-STAT3, STAT3, p-p65 or GAPDH by overnight incubation at $4{ }^{\circ} \mathrm{C}$, followed by chemiluminescent detection according to the manufacturer's instructions.

\section{Mouse tumor model}

MSCs were pretreated with IFN $\gamma$ and TNF $\alpha$, or IFN $\gamma$, TNF $\alpha$ and IL-17A with or without $10 \mu \mathrm{g} / \mathrm{ml}$ PD-L1 antibodies. 2 days later, cells were digested, washed and harvested for tumor model. Each mouse was injected with
$2.5 \times 10^{5}$ B16F0 in $100 \mu \mathrm{l}$ PBS subcutaneously with or without pretreated MSCs $\left(1 \times 10^{5}\right)$. Mice were observed daily. On day 16 after tumor cell administration, the resultant tumors were then excised and weighed. Each experimental group included at least five mice.

\section{Statistical analysis}

Data are presented as mean \pm SEM. Statistical significance was assessed using unpaired two-tailed Student's t-test, ${ }^{*} \mathrm{p}<0.05,{ }^{* * *} \mathrm{p}<0.01$, or Log-Rank test in survival experiment: ${ }^{* * *} \mathrm{p}<0.01,{ }^{* * * *} \mathrm{p}<0.001$.

\section{Abbreviations \\ IFNY: Interferon-y; LL-17: Interleukin-17; iNOS: Inducible nitric oxide synthase; MSCs: Mesenchymal stem cells; NO: Nitric oxide; PD-L1: Programmed death- ligand 1; SMT: S-methyl-isothiourea; SNAP: S-nitroso-N-acetyl-penicillamine; STAT3: Signal transducer and activator of transcription 3; TNFa: Tumor necrosis factor-a.}

\section{Acknowledgements}

This work was supported by grants from the Scientific Innovation Project of the Chinese Academy of Science (XDA 01040107 and XDA 01040110), the Programs of National Natural Science of China (81330046), the Ministry of Science and Technology of China (2015CB964400), the External Cooperation Program of BIC, Chinese Academy of Sciences (GJHZ201307), Shanghai Municipal Key Projects of Basic Research (12JC1409200), Shanghai Rising-Star Program (14QA1404200). The Child Health Institute of New Jersey is supported by a grant from the Robert Wood Johnson Foundation (Grant Number 67038).

\section{Authors' contributions}

SW design and performed the experiment, analyzed the data and prepared the manuscript. GW, LZ, KL helped conducted experiment. FL managed mouse breeding. KC, YW and YS lead the project and wrote the manuscirpt with first author. All authors read and approved the final manuscript.

\section{Funding}

Funding was provided by National Key R\&D program of China

(2018YFA0107500, 2018YFC1704300), the MAECI Italy-China Science and Technology Cooperation (\#PGR00961), National Natural Science of China Programs (81571612, 81530043, 31601106, 31771641, 81861138015), the Scientific Innovation Project of the Chinese Academy of Science (XDA16020403).

Availability of data and materials

Please contact the corresponding author for data on reasonable request.

Competing interests

The authors declare that they have no competing interests.

\section{Author details}

${ }^{1}$ Key Laboratory of Stem Cell Biology, Shanghai Jiao Tong University School of Medicine (SJTUSM) \& Shanghai Institutes for Biological Sciences (SIBS), Academy of Sciences (CAS), Shanghai 200031, China. ${ }^{2}$ CAS Key Laboratory of Tissue Microenvironment and Tumor, Shanghai Institute of Nutrition and Health, Shanghai Institutes for Biological Sciences, University of Chinese Academy of Sciences, Chinese Academy of Sciences, Shanghai 200031, China. ${ }^{3}$ The First Affiliated Hospital of Soochow University, State Key Laboratory of Radiation Medicine and Protection, Institutes for Translational Medicine, Soochow University, Suzhou 215123, China.

Received: 29 December 2019 Accepted: 13 May 2020

Published online: 26 May 2020 


\section{References}

1. Rouvier E, Luciani MF, Mattei MG, Denizot F, Golstein P. CTLA-8, cloned from an activated $T$ cell, bearing AU-rich messenger RNA instability sequences, and homologous to a herpesvirus saimiri gene. J Immunol. 1993:150(12):5445-56.

2. Harrington LE, Hatton RD, Mangan PR, Turner H, Murphy TL, Murphy KM, Weaver CT. Interleukin 17-producing CD4 + effector T cells develop via a lineage distinct from the Thelper type 1 and 2 lineages. Nat Immunol. 2005:6(11):1123-32

3. Park H, Li Z, Yang XO, Chang SH, Nurieva R, Wang YH, Wang Y, Hood L, Zhu $Z$, Tian $Q$, et al. A distinct lineage of CD4 T cells regulates tissue inflammation by producing interleukin 17. Nat Immunol. 2005;6(11):1133-41.

4. Rodrlguez E, Schetters STT, van Kooyk Y. The tumour glyco-code as a novel immune checkpoint for immunotherapy. Nat Rev Immunol. 2018;18(3):204-11.

5. Spitzer MH, Carmi Y, Reticker-Flynn NE, Kwek SS, Madhireddy D, Martins MM, Gherardini PF, Prestwood TR, Chabon J, Bendall SC, et al. Systemic immunity is required for effective cancer immunotherapy. Cell. 2017;168(3):487-502.e415.

6. Miyahara Y, Odunsi K, Chen W, Peng G, Matsuzaki J, Wang RF. Generation and regulation of human CD4+ IL-17-producing $T$ cells in ovarian cancer. Proc Natl Acad Sci USA. 2008;105(40):15505-10.

7. Sfanos KS, Bruno TC, Maris CH, Xu L, Thoburn CJ, DeMarzo AM, Meeker AK, Isaacs WB, Drake CG. Phenotypic analysis of prostate-infiltrating lymphocytes reveals TH17 and Treg skewing. Clin Cancer Res. 2008;14(11):3254-61.

8. Miossec P, Kolls JK. Targeting IL-17 and TH17 cells in chronic inflammation. Nat Rev Drug Discov. 2012;11(10):763-76.

9. Chung AS, Wu X, Zhuang G, Ngu H, Kasman I, Zhang J, Vernes JM, Jiang Z, Meng YG, Peale FV, et al. An interleukin-17-mediated paracrine network promotes tumor resistance to anti-angiogenic therapy. Nat Med. 2013;19(9):1114-23.

10. Amicarella F, Muraro MG, Hirt C, Cremonesi E, Padovan E, Mele V, Governa V, Han J, Huber X, Droeser RA, et al. Dual role of tumour-infiltrating T helper 17 cells in human colorectal cancer. Gut. 2017;66(4):692-704.

11. Numasaki M, Fukushi J, Ono M, Narula SK, Zavodny PJ, Kudo T, Robbins PD, Tahara H, Lotze MT. Interleukin-17 promotes angiogenesis and tumor growth. Blood. 2003;101(7):2620-7.

12. Charles KA, Kulbe H, Soper R, Escorcio-Correia M, Lawrence T, Schultheis A, Chakravarty P, Thompson RG, Kollias G, Smyth JF, et al. The tumor-promoting actions of TNF-alpha involve TNFR1 and IL-17 in ovarian cancer in mice and humans. J Clin Investig. 2009:119(10):3011-23.

13. Dvorak HF. Tumors: wounds that do not heal. Similarities between tumor stroma generation and wound healing. N Engl J Med. 1986;315(26):1650-9.

14. Shi Y, Du L, Lin L, Wang Y. Tumour-associated mesenchymal stem/ stromal cells: emerging therapeutic targets. Nat Rev Drug Discov. 2017;16(1):35-52.

15. Ren $G$, Zhao X, Wang $Y$, Zhang $X$, Chen $X, X u$ C, Yuan ZR, Roberts Al, Zhang $L$, Zheng B, et al. CCR2-dependent recruitment of macrophages by tumor-educated mesenchymal stromal cells promotes tumor development and is mimicked by TNFalpha. Cell Stem Cell. 2012;11(6):812-24.

16. Lin LY, Du LM, Cao K, Huang Y, Yu PF, Zhang LY, Li FY, Wang Y, Shi YF. Tumour cell-derived exosomes endow mesenchymal stromal cells with tumour-promotion capabilities. Oncogene. 2016;35(46):6038-42.

17. Lin L, Du L. The role of secreted factors in stem cells-mediated immune regulation. Cell Immunol. 2018;326:24-32.

18. Wang Y, Chen X, Cao W, Shi Y. Plasticity of mesenchymal stem cells in immunomodulation: pathological and therapeutic implications. Nat Immunol. 2014:15(11):1009-16.

19. Ren G, Zhang L, Zhao X, Xu G, Zhang Y, Roberts Al, Zhao RC, Shi Y. Mesenchymal stem cell-mediated immunosuppression occurs via concerted action of chemokines and nitric oxide. Cell Stem Cell. 2008;2(2):141-50.

20. Cao W, Cao K, Cao J, Wang Y, Shi Y. Mesenchymal stem cells and adaptive immune responses. Immunol Lett. 2015;168(2):147-53.

21. Yu PF, Huang Y, Han YY, Lin LY, Sun WH, Rabson AB, Wang Y, Shi YF. TNFalpha-activated mesenchymal stromal cells promote breast cancer metastasis by recruiting CXCR2(+) neutrophils. Oncogene. 2017;36(4):482-90.

22. Shi Y, Wang Y, Li Q, Liu K, Hou J, Shao C, Wang Y. Immunoregulatory mechanisms of mesenchymal stem and stromal cells in inflammatory diseases. Nat Rev Nephrol. 2018;14(8):493-507.
23. Du L, Lin L, Li Q, Liu K, Huang Y, Wang X, Cao K, Chen X, Cao W, Li F, et al. IGF-2 preprograms maturing macrophages to acquire oxidative phosphorylation-dependent anti-inflammatory properties. Cell Metab. 2019;29(6):1363-1375.e1368.

24. Han X, Yang Q, Lin L, Xu C, Zheng C, Chen X, Han Y, Li M, Cao W, Cao K, et al. Interleukin-17 enhances immunosuppression by mesenchymal stem cells. Cell Death Differ. 2014;21(11):1758-68.

25. Sharpe AH, Wherry EJ, Ahmed R, Freeman GJ. The function of programmed cell death 1 and its ligands in regulating autoimmunity and infection. Nat Immunol. 2007;8(3):239-45.

26. Latchman YE, Liang SC, Wu Y, Chernova T, Sobel RA, Klemm M, Kuchroo VK, Freeman GJ, Sharpe AH. PD-L1-deficient mice show that PD-L1 on $T$ cells, antigen-presenting cells, and host tissues negatively regulates $T$ cells. Proc Natl Acad Sci USA. 2004;101(29):10691-6.

27. Fife BT, Pauken KE, Eagar TN, Obu T, Wu J, Tang Q, Azuma M, Krummel MF, Bluestone JA. Interactions between PD-1 and PD-L1 promote tolerance by blocking the TCR-induced stop signal. Nat Immunol. 2009:10(11):1185-92

28. Zhou J, Peng H, Li K, Qu K, Wang B, Wu Y, Ye L, Dong Z, Wei H, Sun R, et al. Liver-resident NK cells control antiviral activity of hepatic T cells via the PD-1-PD-L1 axis. Immunity. 2019;50(2):403-417.e404.

29. Sun C, Mezzadra R, Schumacher TN. Regulation and function of the PD-L1 checkpoint. Immunity. 2018;48(3):434-52.

30. Pitt JM, Vetizou M, Daillere R, Roberti MP, Yamazaki T, Routy B, Lepage P, Boneca IG, Chamaillard M, Kroemer G, et al. Resistance mechanisms to immune-checkpoint blockade in cancer: tumor-intrinsic and -extrinsic factors. Immunity. 2016;44(6):1255-69.

31. Baumeister SH, Freeman GJ, Dranoff G, Sharpe AH. Coinhibitory pathways in immunotherapy for cancer. Annu Rev Immunol. 2016;34:539-73.

32. Callahan MK, Postow MA, Wolchok JD. Targeting T cell co-receptors for cancer therapy. Immunity. 2016:44(5):1069-78.

33. Luz-Crawford P, Noel D, Fernandez X, Khoury M, Figueroa F, Carrion F, Jorgensen C, Djouad F. Mesenchymal stem cells repress Th17 molecular program through the PD-1 pathway. PLoS ONE. 2012;7(9):e45272.

34. Sheng H, Wang Y, Jin Y, Zhang Q, Zhang Y, Wang L, Shen B, Yin S, Liu W, Cui $L$, et al. A critical role of IFNgamma in priming MSC-mediated suppression of T cell proliferation through up-regulation of B7-H1. Cell Res. 2008;18(8):846-57.

35. Augello A, Tasso R, Negrini SM, Amateis A, Indiveri F, Cancedda R, Pennesi G. Bone marrow mesenchymal progenitor cells inhibit lymphocyte proliferation by activation of the programmed death 1 pathway. Eur J Immunol. 2005:35(5):1482-90.

36. Zhao Q, Xiao X, Wu Y, Wei Y, Zhu LY, Zhou J, Kuang DM. Interleukin-17-educated monocytes suppress cytotoxic T-cell function through B7-H1 in hepatocellular carcinoma patients. Eur J Immunol. 2011;41(8):2314-22.

37. Dong $\mathrm{H}$, Chen L. B7-H1 pathway and its role in the evasion of tumor immunity. J Mol Med. 2003;81(5):281-7.

38. Kondo A, Yamashita T, Tamura H, Zhao W, Tsuji T, Shimizu M, Shinya E, Takahashi H, Tamada K, Chen L, et al. Interferon-gamma and tumor necrosis factor-alpha induce an immunoinhibitory molecule, B7-H1, via nuclear factor-kappaB activation in blasts in myelodysplastic syndromes. Blood. 2010;116(7):1124-31

39. Xiao M, Wang C, Zhang J, Li Z, Zhao X, Qin Z. IFNgamma promotes papilloma development by up-regulating Th17-associated inflammation. Cancer Res. 2009:69(5):2010-7.

40. Stephens JM, Carter BZ, Pekala PH, Malter JS. Tumor necrosis factor alpha-induced glucose transporter (GLUT-1) mRNA stabilization in 3T3-L1 preadipocytes. Regulation by the adenosine-uridine binding factor. J Biol Chem. 1992;267(12):8336-41.

41. Marzec M, Zhang Q, Goradia A, Raghunath PN, Liu X, Paessler M, Wang HY, Wysocka M, Cheng M, Ruggeri BA, et al. Oncogenic kinase NPM/ALK induces through STAT3 expression of immunosuppressive protein CD274 (PD-L1, B7-H1). Proc Natl Acad Sci USA. 2008;105(52):20852-7.

42. Wolfle SJ, Strebovsky J, Bartz H, Sahr A, Arnold C, Kaiser C, Dalpke AH, Heeg K. PD-L1 expression on tolerogenic APCs is controlled by STAT-3. Eur J Immunol. 2011:41(2):413-24.

43. Durand-Panteix S, Farhat M, Youlyouz-Marfak I, Rouaud P, Ouk-Martin C, David A, Faumont N, Feuillard J, Jayat-Vignoles C. B7-H1, which represses EBV-immortalized B cell killing by autologous T and NK cells, is oppositely regulated by c-Myc and EBV latency III program at both mRNA and secretory lysosome levels. J Immunol. 2012;189(1):181-90. 
44. Ivashkiv LB. IFNgamma: signalling, epigenetics and roles in immunity, metabolism, disease and cancer immunotherapy. Nat Rev Immunol. 2018;18(9):545-58.

45. Dostert C, Grusdat M, Letellier E, Brenner D. The TNF family of ligands and receptors: communication modules in the immune system and beyond. Physiol Rev. 2019;99(1):115-60.

46. Lundberg JO, Weitzberg E, Gladwin MT. The nitrate-nitrite-nitric oxide pathway in physiology and therapeutics. Nat Rev Drug Discov. 2008;7(2):156-67.
47. Radi R. Protein tyrosine nitration: biochemical mechanisms and structural basis of functional effects. Acc Chem Res. 2013;46(2):550-9.

\section{Publisher's Note}

Springer Nature remains neutral with regard to jurisdictional claims in published maps and institutional affiliations.
Ready to submit your research? Choose BMC and benefit from:

- fast, convenient online submission

- thorough peer review by experienced researchers in your field

- rapid publication on acceptance

- support for research data, including large and complex data types

- gold Open Access which fosters wider collaboration and increased citations

- maximum visibility for your research: over $100 \mathrm{M}$ website views per year

At BMC, research is always in progress.

Learn more biomedcentral.com/submissions 